PROCEEDINGS OF THE

AMERICAN MATHEMATICAL SOCIETY

Volume 125, Number 10, October 1997, Pages 2997-3001

S 0002-9939(97)03937-3

\title{
THE VALUE DISTRIBUTION OF THE HYPERBOLIC GAUSS MAP
}

\author{
ZU-HUAN YU
}

(Communicated by Christopher Croke)

\begin{abstract}
In this paper, we investigate the hyperbolic Gauss map of a complete CMC-1 surface in $H^{3}(-1)$, and prove that it cannot omit more than four points unless the surface is a horosphere.
\end{abstract}

\section{INTRODUCTION}

In minimal surface theory, the value distribution of the Gauss map has been studied for a long time. An early result is the classical Bernstein Theorem: Any complete minimal graph in $R^{3}$ is a plane. Replacing the graph by some geometric conditions, R. Osserman [3] showed that if the complete minimal surface is nonflat, then the Gauss map cannot omit a set of positive logarithmic capacity, which answered a conjecture of Nirenberg. Afterwards, it was generalized by F. Xavier [8] that the Gauss map of such a surface can omit at most six points. In 1989, H. Fujimoto [2] proved the best result finally that the Gauss map of the nonflat complete minimal surface in $R^{3}$ can omit at most four points.

It is natural to consider the similar properties of minimal surfaces in $H^{3}$. An interesting feature is that there exists a family of absolutely area-minimizing hypersurfaces in $H^{n}$ and only one of them is totally geodesic (see [7]). The question seems to be how to raise an adequate Bernstein problem in hyperbolic space. Professor Y. L. Xin pointed out to me that the striking work done by R. Bryant [1] supplies a framework to solve the Bernstein problem in hyperbolic space.

In this paper, we shall be concerned with the surfaces in hyperbolic space of constant mean curvature one. We abbreviate "constant mean curvature one" by CMC-1. These surfaces share many properties with minimal surfaces in $R^{3}$. They possess the "Weierstrass representation" in terms of holomorphic data. This formula was discovered by R. Bryant [1]. Many other properties may be found in papers by M. Umhara and K. Yamada ([4], [5]). Here we try to investigate the hyperbolic analogue of the Gauss map. It is a natural question how the values of the hyperbolic Gauss map distribute. Using Bryant's representation formula we are able to answer this question as follows.

Theorem. The hyperbolic Gauss map of nonflat complete CMC-1 surfaces in $H^{3}(-1)$ can omit at most four points.

Received by the editors November 1, 1995 and, in revised form, April 2, 1996

1991 Mathematics Subject Classification. Primary 53A10; Secondary 53C42.

Key words and phrases. Constant mean curvature, hyperbolic space, Gauss map.

Partially supported by NNSFC and SFECC. 
Remark 1. For the above mentioned surfaces in the Theorem, if their hyperbolic Gauss map omits more than four points, they have to be horospheres.

Remark 2. It is easily shown that there exist complete CMC-1 surfaces in $H^{3}(-1)$ whose hyperbolic Gauss map can omit $k$ points, $k=0,1,2,3,4$.

\section{Preliminaries}

We list the main results which will be used in this paper. For details readers are referred to [1]. Let $L^{4}$ denote Minkowski space with coordinates $x_{0}, x_{1}, x_{2}, x_{3}$, and let the inner product be given by the quadratic differential form $-\left(d x_{0}\right)^{2}+\left(d x_{1}\right)^{2}+$ $\left(d x_{2}\right)^{2}+\left(d x_{3}\right)^{2}$. We set

$$
H^{3}(-1)=\left\{x \in L^{4} \mid\langle x, x\rangle=-1, x_{0}>0\right\}
$$

and give $H^{3}(-1)$ the induced metric as a space-like hypersurface of $L^{4}$, which is complete with constant sectional curvature -1 .

Now we identify $L^{4}$ with the space $\operatorname{Her}(2)$ of $(2 \times 2)$-Hermitian symmetric matrices, by identifying $\left(x_{0}, x_{1}, x_{2}, x_{3}\right)$ with the matrix

$$
\left(\begin{array}{cc}
x_{0}+x_{3} & x_{1}+i x_{2} \\
x_{1}-i x_{2} & x_{0}-x_{3}
\end{array}\right) .
$$

The complex Lie group $S L(2, C)$ acts on $L^{4}$ naturally by the representation

$$
g \cdot v=g v g^{*}
$$

where $g^{*}=\bar{g}^{t}, g \in S L(2, C), v \in \operatorname{Her}(2)$. Clearly we have $\langle v, v\rangle=-\operatorname{det}(v)$, thus this action preserves $\langle$,$\rangle , and P S L(2 C)=S L(2, C) /\{ \pm I\}$ is viewed as the identity component of $S O(3,1)$. So we can represent

$$
H^{3}(-1)=\left\{a \cdot a^{*} \mid a \in S L(2, C)\right\} .
$$

Let $f: M \rightarrow H^{3}(-1)$ be an oriented CMC-1 surface in $H^{3}(-1)$. There is a null immersion from Riemannian surface $M$ to $S L(2, C)$,

$$
\begin{gathered}
F: M \rightarrow S L(2, C), \\
F=\left(\begin{array}{ll}
F_{1} & F_{2} \\
F_{3} & F_{4}
\end{array}\right), \quad \operatorname{det}(F)=1, \\
\operatorname{det}\left(F^{-1} d F\right)=0,
\end{gathered}
$$

such that

where $F^{*}=\bar{F}^{t}$. We set

$$
f=F \cdot F^{*}
$$

$$
F^{-1} d F=\left(\begin{array}{cc}
g & -g^{2} \\
1 & -g
\end{array}\right) h d z,
$$

where $g, h$ are meromorphic and holomorphic functions on $M$, respectively. The induced metric may be written as

$$
d s^{2}=|h|^{2}\left(1+|g|^{2}\right)^{2}|d z|^{2} .
$$

We now describe the hyperbolic Gauss map of the surface $f: M \rightarrow H^{3}(-1)$. At each point $e_{0}=f(m)(m \in M)$, there exists an oriented geodesic of $H^{3}(-1)$, passing through $e_{0}$ and its tangent vector at point $e_{0}$ is the normal vector of $f(M)$. This oriented geodesic meets the ideal boundary of $H^{3}(-1)$ at a point. Hence one 
gets a map from $M$ to the ideal boundary $C P^{1}$, denoted by $G$. It is called the hyperbolic Gauss map. By means of the holomorphic representation, $G$ can be read as

$$
\begin{gathered}
G: M \rightarrow C P^{1}, \\
G(z)=\left[d F_{1}, d F_{3}\right] .
\end{gathered}
$$

\section{Proof of the Theorem}

For convenience, let us suppose that $M$ is a simply connected Riemannian surface, otherwise, choose the universal cover of $M$. Let $f: M \rightarrow H^{3}(-1)$ be a conformal CMC-1 surface. The holomorphic representation $F$ is a null immersion

$$
F: M \rightarrow S L(2, C) .
$$

Clearly, the inverse matrix $F^{-1}$ can be viewed as a holomorphic null immersion from $M$ into $S L(2, C)$

$$
F^{-1}: M \rightarrow S L(2, C)
$$

Setting $f^{-1}=F^{-1} \cdot\left(F^{-1}\right)^{*}$, and using Theorem A in [1], $f^{-1}$ is a new CMC-1 surface

$$
f^{-1}: M \rightarrow H^{3}(-1)
$$

It is called the inverse surface of $f(M)$. Now defining $G_{0}, G_{1}$ by $G_{0}=F_{1} g+F_{2}$ and $G_{1}=F_{3} g+F_{4}$, from (1.2) we get

$$
\left(\begin{array}{l}
G_{0} \\
G_{1}
\end{array}\right)=\frac{1}{h}\left(\begin{array}{l}
d F_{1} \\
d F_{3}
\end{array}\right)=F\left(\begin{array}{l}
g \\
1
\end{array}\right)=\left(\begin{array}{ll}
F_{1} & F_{2} \\
F_{3} & F_{4}
\end{array}\right)\left(\begin{array}{l}
g \\
1
\end{array}\right) .
$$

Noting (1.4), it is clear that $\left(\begin{array}{l}G_{0} \\ G_{1}\end{array}\right)$ are the homogeneous coordinates of the hyperbolic Gauss map $G$. By (1.2) and a simple calculation we have

$$
F d F^{-1}=\left(\begin{array}{cc}
G & -G^{2} \\
1 & -G
\end{array}\right) H d z
$$

where $G=\frac{G_{0}}{G_{1}}, H=-G_{1}^{2} h$. The metric of surface $f^{-1}(M)$ may be expressed in terms of data $(G, H)$ as

$$
d s^{2}=|H|^{2}\left(1+|G|^{2}\right)^{2}|d z|^{2} .
$$

Note that the meromorphic function $G$ in (2.5) and (2.6) is the nonhomogeneous coordinate of the hyperbolic Gauss map $G$ of $f(M)$. Since $\left(f^{-1}\right)^{-1}=f$, then the meromorphic function $g$ in (1.2) and (1.3) is the nonhomogeneous coordinate of the hyperbolic Gauss map of $f^{-1}(M)$.

In what follows, we prove a proposition which is crucial in the proof of the Theorem.

Proposition. The surface $f: M \rightarrow H^{3}(-1)$ is complete if and only if the inverse surface $f^{-1}: M \rightarrow H^{3}(-1)$ is complete.

Proof. Since $\left(f^{-1}\right)^{-1}=f$, we only need to certify the necessary condition; it is enough to show that if $f^{-1}$ is not complete, then $f$ is not yet complete. Suppose that $f^{-1}$ is not complete; then there exists a divergent curve $\gamma$ starting from some 
point $p_{0} \in M$ and the length of $\gamma_{1}=f^{-1}(\gamma)$ is finite. Without loss generality, we set $f^{-1}\left(p_{0}\right)=(1,0,0,0)$,

$$
\int_{\gamma_{1}} d s=\int_{\gamma}|H|\left(1+|G|^{2}\right)|d z|<+\infty .
$$

Therefore the curve $\gamma_{1}$ is bounded in $H^{3}(-1)$, namely there is a geodesic sphere with center $(1,0,0,0)$ which contains this curve. Otherwise, there should exist a sequence of points $p_{1}, p_{2}, \ldots, p_{n}, \ldots$ on $\gamma$, and $\operatorname{dist}\left(f^{-1}\left(p_{n}\right), f^{-1}\left(p_{0}\right)\right)$ diverges to $+\infty$. On the other hand, $\operatorname{dist}\left(f^{-1}\left(p_{n}\right), f^{-1}\left(p_{0}\right)\right)$ is less than the arc length of $\gamma_{1}$ between $f^{-1}\left(p_{n}\right)$ and $f^{-1}\left(p_{0}\right)$. So we conclude that the length of $\gamma_{1}$ is infinite. This contradicts the hypothesis. Since the Minkowski coordinates of $\gamma_{1}$ are bounded, and noting the relationship between $L^{4}$ and $\operatorname{Her}(2)$, then the matrix $F^{-1}(\gamma)$ is bounded. So is $F(\gamma)$, which means

$$
\sup _{\gamma}\left|F_{i}\right| \leq N \quad(i=1,2,3,4)
$$

where $N$ is a positive number. From (2.4) we get

$$
\left(\begin{array}{l}
g \\
1
\end{array}\right)=F^{-1}\left(\begin{array}{l}
G_{0} \\
G_{1}
\end{array}\right)
$$

and

$$
1+|g|^{2} \leq 4 N^{2}\left(\left|G_{0}\right|^{2}+\left|G_{1}\right|^{2}\right)
$$

On the other hand, $f(\gamma)$ is a curve on the surface $f(M)$ which is divergent to the boundary, and the length can be estimated as follows:

$$
\begin{aligned}
\int_{\gamma}|h|\left(1+|g|^{2}\right)|d z| & \leq \int_{\gamma}|h| 4 N^{2}\left(\left|G_{0}\right|^{2}+\left|G_{1}\right|^{2}\right)|d z| \\
& =4 N^{2} \int_{\gamma}\left|h G_{1}^{2}\right|\left(1+|G|^{2}\right)|d z| \\
& =4 N^{2} \int_{\gamma}|H|\left(1+|G|^{2}\right)|d z|<+\infty .
\end{aligned}
$$

This implies that the surface $f(M)$ is not complete. The proposition is proved entirely.

Remark 3. The definition of the inverse surface (= dual surface) is also given in a preprint of M. Umehara and K. Yamada [6], in which the completeness of the inverse surface is shown under the assumption that all ends are regular.

Appealing to the proposition above, one can easily give the proof of the Theorem.

Proof of the Theorem. In previous arguments, the hyperbolic Gauss map $G$ of $f(M)$ is the meromorphic function $G$ in the metric of $f^{-1}(M)$. Since $f^{-1}(M)$ is complete, we get a complete minimal immersion in $R^{3}$ by the Weierstrass data $(G, H)$ and Weierstrass formula, denoting it by $f^{\prime}$,

$$
\begin{gathered}
f^{\prime}: M \rightarrow R^{3} \\
f^{\prime}(z)=\operatorname{Re}\left(\frac{1}{2} \int\left(1-G^{2}\right) H d z, \frac{i}{2} \int\left(1+G^{2}\right) H d z, \int G H d z\right) .
\end{gathered}
$$


The metric of this minimal immersion is

$$
d s^{2}=|H|^{2}\left(1+|G|^{2}\right)^{2}|d z|^{2}
$$

and the Gauss map is $G$. By applying the Fujimoto theorem to the surface $f^{\prime}(M)$, one obtains that $G$ must be constant if it omits more than four points. Now, if $G$ is constant, from

$$
G=\frac{d F_{1}}{d F_{3}}=\frac{d F_{2}}{d F_{4}}
$$

we have

$$
\begin{aligned}
& F_{1}=G F_{3}+C_{1}, \\
& F_{2}=G F_{4}+C_{2},
\end{aligned}
$$

where $C_{1}, C_{2}$ are constant. On the other hand, by a direct computation we get

$$
g=\frac{G F_{4}-F_{2}}{F_{1}-G F_{3}} .
$$

Combining (2.11) and (2.12) gives

$$
g=-\frac{C_{2}}{C_{1}}
$$

Then $g$ is constant. Consequently, $f(M)$ is flat and a horosphere. We complete the proof of the Theorem.

\section{ACKNOWLEDGMENT}

I would like to thank Professor Y. L. Xin for his concrete guidance, Professor H. $\mathrm{S}$. $\mathrm{Hu}$ for her encouragement, and the referee for sending me the preprint [6] and helpful suggestions.

\section{REFERENCES}

[1] R. L. Bryant, Surfaces of mean curvature one in hyperbolic space, Asterisque 154-155 (1987), 321-347. CMP 20:16

[2] H. Fujimoto, Modified defect relations for the Gauss map of minimal surfaces, J. Diff. Geo. 29 (1989), 245-262. MR 89m:53012

[3] R. Osserman, Minimal surfaces in the large, Comment. Math. Helv. 35 (1961), 65-76. MR 23:A576

[4] M. Umehara and K. Yamada, Complete surfaces of constant mean curvature-1 in the hyperbolic 3-space, Ann. of Math. 137 (1993), 611-638. MR 94c:53015

[5] M. Umehara and K. Yamada, A parametrization of the Weierstrass formulae minimal surfaces in $R^{3}$ into the hyperbolic 3-space, J. Reine Angew. Math. 432 (1992), 93-116. MR 94e:54004

[6] _ A duality on CMC-1 surfaces in hyperbolic space, and a hyperbolic analogue of the Osserman inequality, (preprint).

[7] S. P. Wang and S. Walter Wei, Bernstein conjecture in hyperbolic geometry, Seminar on minimal submanifolds, Ann. Math. Studies 103, Princeton Univ. Press, 1983. MR 86m:53076

[8] F. Xavier, The Gauss map of a complete nonflat minimal surface cannot omit 7 points of the sphere, Ann. of Math. (2) 133 (1981), 211-214. MR 83h:53016 CHINA

Institute of Mathematics, Fudan University, Shanghai 200433, People's Republic of

Current address: Fundamental Department, Jiaozuo Institute of Technology, Jiaozuo 454159, Henan Province, People's Republic of China 